\title{
Observation of Participator Auger Decay following Valence Photoionization with Excitation
}

\author{
U. Becker, R. Wehlitz, O. Hemmers, B. Langer, and A. Menzel \\ Institut für Strahlungs - und Kernphysik, Technische Universität Berlin, D-1000 Berlin 12, West Germany
} (Received 31 May 1989)

\begin{abstract}
"Complete" valence photoelectron spectra of neon and argon have been measured at several photon energies between 50 and $150 \mathrm{eV}$. Besides a symmetric shake-off energy spectrum as anticipated theoretically many unexpected electron lines with fixed kinetic energy were observed, particularly in the lowenergy part of the spectra. These lines are identified as due to participator Auger transitions from inner-valence satellite states. The consequence of the measured strengths of the valence Auger transitions regarding the common interpretation of valence double-ionization rates in terms of shake-off processes is discussed.
\end{abstract}

PACS numbers: $32.80 . \mathrm{Fb}, 32.80 . \mathrm{Hd}, 79.60 .-\mathrm{i}$

Auger decay ${ }^{1}$ is one of the most characteristic aspects of an inner-shell vacancy distinguishing these vacancies in a phenomenological point of view from those in the valence shell. This is because the energy gained by the vacancy-filling recombination process must be large enough to remove an outer electron from the atom or molecule, a condition usually fulfilled only by core-level vacancies. A comprehensive review of Auger-electron spectrometry of core levels of atoms is given by Mehlhorn. ${ }^{2}$

Valence-shell holes do not decay via Auger transitions because insufficient excess energy. In practice, this point is somewhat academic, because ionization processes are in many cases accompanied by additional excitation of an outer electron to an unoccupied level leaving the remaining ion in an excited state. ${ }^{3-5}$ In this case, the excess energy necessary for Auger decay may be sufficiently small that Auger decay of valence vacancies becomes possible. Because of the necessity of the excited electron to participate in such a decay, we call this process participator Auger decay. Clear evidence for participator Auger transitions following inner-shell ionization was reported for the first time by Krause, Carlson, and Moddemann $^{6}$ in their presentation of a "complete" Auger spectrum of neon obtained with high energy resolution. In this study all the observed lines are classified in groups according to their originating processes, one of them being Auger transitions from satellite states with the excited electron participating in the transition, the so-called $C \beta$ lines. The participator Auger lines have higher kinetic energy than their pertinent diagram lines because the same energy that the photoelectron loses by the additional excitation is gained by the corresponding Auger electron in the subsequent deexcitation process. It is this same mechanism as in the Auger decay of corehole vacancies which enables valence vacancy states to decay also via Auger transitions. A schematic representation of the processes discussed above is given in Fig. 1 (a) showing the $2 s$ ionization of neon without any additional excitation: Recombination of the $2 s$ vacancy via "normal" Auger decay is energetically not allowed. In contrast Fig. 1(b) shows how a $2 s$ vacancy state populated by simultaneous ionization and excitation may decay via the "participator" Auger transition. Up to now photoelectron studies of valence-shell ionization showed little evidence for the existence of such Auger transitions. Kossmann et al. ${ }^{7}$ mentioned some weak features in their high-resolution $3 s$ photoelectron spectrum of argon which they designated tentatively in part to nonradiative decay of $3 s$ satellite states, but they did not consider these features further in their study. It was expected that two-electron processes in general and participator Auger channels particularly are of minor importance compared to other ionization and decay mechanisms, the latter substantiated by inner-shell studies of Krause, Carlson, and Moddemann ${ }^{6}$ showing that participator transitions are reduced by an order of magnitude compared to the corresponding spectator transitions.

Photoelectron spectra of the valence subshells of rare gases are the ones studied most elaborately. In this Letter we report on a series of complete photoelectron spectra of neon and argon from threshold up to the $2 s$ or $3 s$ photoline, respectively, taken at different excitation energies. In contrast to expectations we show here for
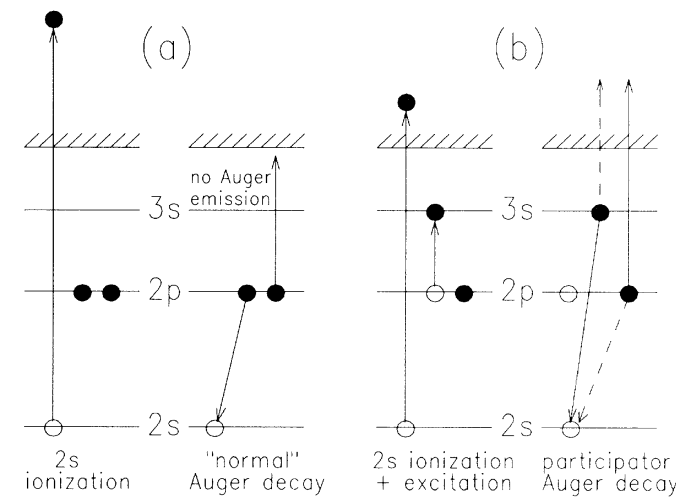

FIG. 1. Schematic level and transition diagram of neon $2 s$ photoionization (a) without and (b) with accompanying excitation along with subsequent recombination processes. 
the first time spectra of ejected electrons with many distinct participator Auger lines, particularly in the scarcely investigated low-energy part of these spectra. The characteristic feature of the participator Auger lines is, completely analogous to normal Auger lines, that they appear at the same kinetic energy for all photon energies above threshold. This behavior is not restricted to certain excitation energies, thus distinguishing the observed process from autoionization.

We have recorded valence photoelectron spectra of neon and argon in the photon energy range from 50 to $150 \mathrm{eV}$ with main emphasis on the low-energy part of each spectrum. The $2 s$ and $3 s$ satellites in neon and argon, respectivley, were assigned according to the designation given by Svensson et al. ${ }^{8}$ Additional interest in the registration of nearly background-free complete photoelectron spectra was stimulated by the possibility to verify the theoretically predicted symmetric energy distribution of shake-off electrons at the low-energy side for the first time experimentally.

The experiments were performed at the Hamburger

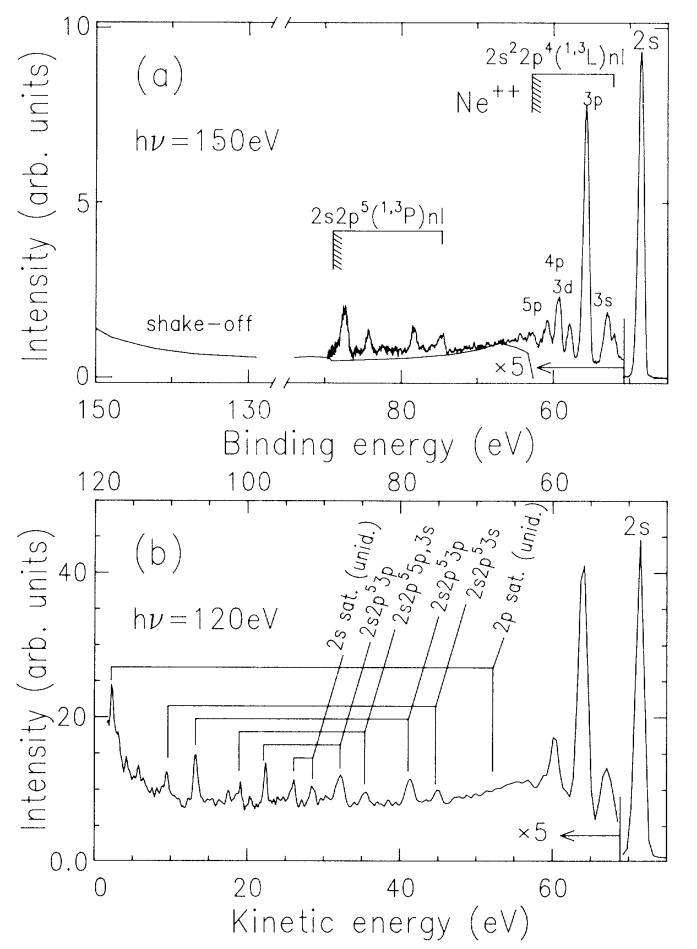

FIG. 2. Spectrum of ejected electrons in neon (a) from 70 $\mathrm{eV}$ and (b) from threshold up to the $3 s$ photoline measured under the quasimagic angle. The cut spectrum displayed in (a) was taken at a photon energy of $150 \mathrm{eV}$ whereas the complete spectrum was taken at $120 \mathrm{eV}$ causing the difference in binding-energy scale, particularly regarding the shake-off energy distribution. The solid curve represents a theoretical shake-off spectrum (Refs. 13 and 14) adjusted to the near-threshold intensity of our experimental spectrum, the two thresholds being depicted.
Synchrotronstrahlungslabor HASYLAB using photons from the $5.6-\mathrm{m}$ toroidal grating monochromator during single-bunch operation of the electron storage ring DORIS II. The large time window of this operational mode suggests time-of-flight analysis ${ }^{9}$ in order to record complete photoelectron spectra. Our time-of-flight spectrometer having a flight pass of $679 \mathrm{~mm}$ is fairly well adjusted to perform high-resolution time-of-flight analysis down to threshold within the given time window of 960 nsec. The characteristic features of our spectrometer system and the beam line employed are described in some detail in former publications. ${ }^{10,11}$ Here it is worthwhile to mention that a significant decrease in dark pulses to 0.5 integral count per spectrum and second made it possible to determine and analyze shake-off intensities and energy distributions unambiguously, which was the original purpose of this investigation.

Most of the photoelectron spectra taken in earlier experiments were limited to a certain fraction of the spectrum: in the case of neon valence photoionization, for example, to the $2 p$ satellites, and in the most extended cases, to the high-energy part of the shake-off spectrum having on top of it the most prominent $2 s$ satellites. ${ }^{12}$ Figure 2(a) shows such an incomplete Ne valence satellite spectrum as taken by our time-of-flight spectrometer at $h v=150 \mathrm{eV}$ using retardation to achieve better resolution, but sacrificing at the same time the low-kineticenergy part of the spectrum. This was, however, expected to be a mirror image of the high-energy shake-off spectrum $^{13,15,16}$ with little additional structure. The experimental proof of the complete shake-off energy distribution was performed by applying a small acceleration voltage of $2 \mathrm{~V}$ instead of retardation; the surprising result is shown in Fig. 2(b). Whereas, on the one hand, the recorded spectrum confirms the theoretical predictions concerning the shake-off energy distribution ${ }^{13,14}$ in a very nice way, our results are, on the other hand, in

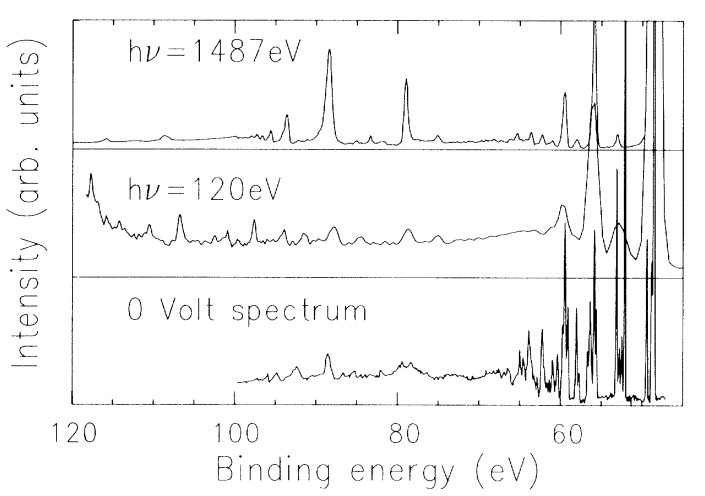

FIG. 3. Comparison of the complete spectrum from Fig. 2(b) with a photoelectron spectrum taken at high photon energy (Al Ka) (Ref. 8) and with a zero-volt electron scan composed from two different measurements (Ref. 17) displaying threshold intensities of satellite lines. 
marked contrast to the expectation of a structureless continuum. The low-energy part of the complete photoelectron spectrum is highly structured exhibiting many distinct lines. In order to identify and classify these newly observed lines we compared our spectrum with highresolution photoelectron spectra taken at high photon energies $(h v-1487 \mathrm{eV})^{8}$ and with a zero-volt electron scan taken across the energy region being considered. ${ }^{17}$ Figure 3 shows these three spectra on a common energy scale. It is evident that many of the lines exhibited in our spectrum have no counterpart in the two other spectra. The resulting assumption that these lines are Auger lines which could not appear in the high-energy and zero-volt electron spectra was verified by comparing different spectra taken at different excitation energies. Figure 4 shows such a series of spectra for (a) neon and (b) argon. It is obvious from this figure that in contrast to most of the moving photoelectron lines there are some lines staying at fixed kinetic energy independent of the excitation energy, a clear indication for Auger lines. More specifically it could be shown that most of the stronger Auger transitions belong to specific shake-up satellites accompanying inner-valence photoionization of neon or argon, respectively; few lines seem to be related to $2 p$ satellites for energy reasons. Figure $2(\mathrm{~b})$ shows in

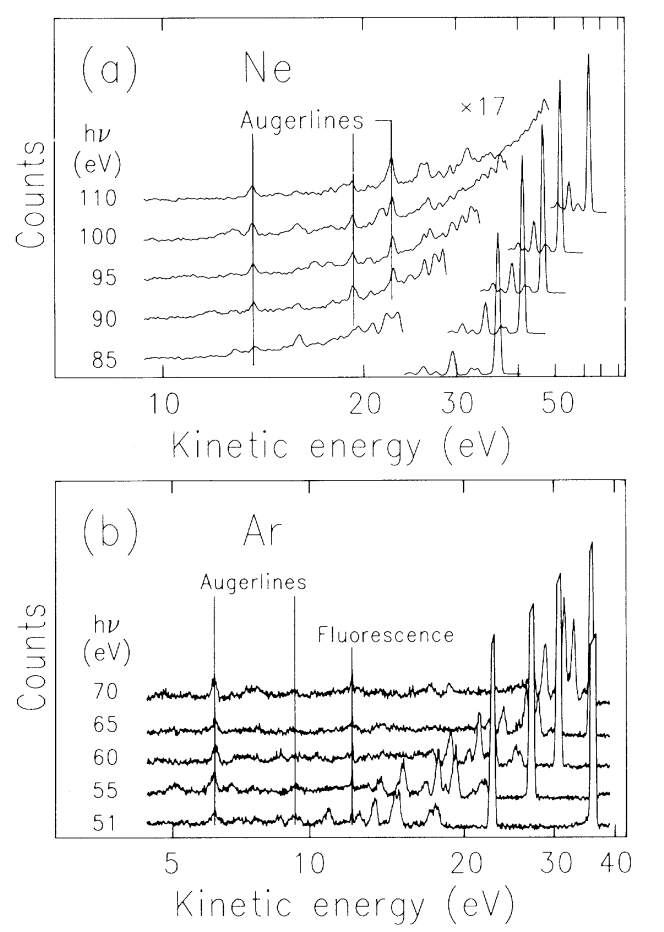

FIG. 4. Series of spectra of ejected electrons in (a) neon and (b) argon having each spectra measured at different photon energies. Some of the Auger lines are depicted with connecting lines to guide the eye; the peak in (b) labeled by fluorescence comprises both Rayleigh scattering of the incoming photons and fluorescence intensity from excited ionic states. more detail how the different Auger lines and shake-up transitions correspond to each other. The strongest Auger transitions depicted in this figure are those to ${ }^{1} D$ states similar to normal Auger decay subsequent to inner-shell ionization. Some of the $2 s$ and $2 p$ satellites supposedly related to certain Auger transitions are still unidentified. Further studies will be necessary to identify and relate all observed lines of ejected electrons to each other.

One essential consequence of the expectation of negligible valence Auger intensity was the interpretation of valence double ionization as purely due to valence shake-off transitions. All theoretical shake-off-calculations ${ }^{13,14}$ were compared to experimental results on the basis of this assumption or, more specifically, but the other way around, all ion yield measurements concerning valence photoionization were compared to shake-off calculations. Our results on valence Auger transitions require a reconsideration of these earlier studies.

Figure 5 displays our partial cross sections for shakeoff and Auger transitions along with photoionization cross sections for multiple ionization of neon. Two different sets of measurements are compared. The ion yield measurements using a magnetic field mass spectrome$\operatorname{ter}^{20}$ show good agreement between the doubleionization yield and the sum of our shake-off and Auger line intensity, while, in the high-energy limit, the separated shake-off intensity is $30 \%$ lower than the corresponding double-ionization yield. However, the latter

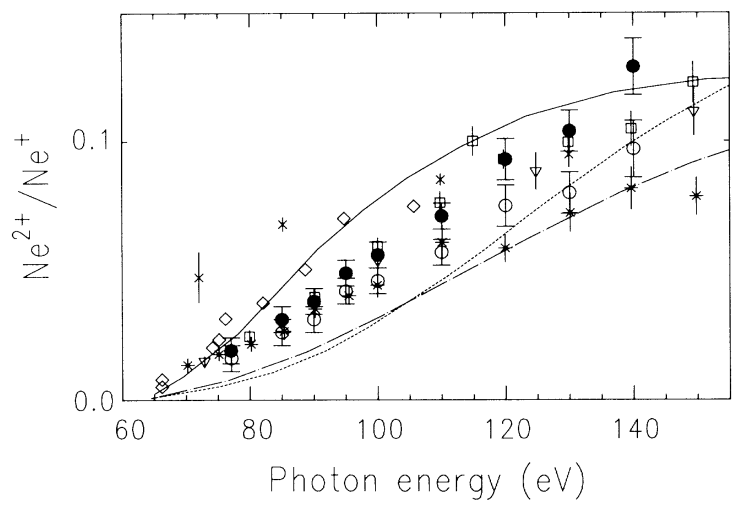

FIG. 5. Separated shake-off intensity (open circles) and the sum of valence shake-off and Auger intensity (filled circles) of this work compared to different ion yield measurements and calculations for the double- to single-ionization yield of neon. The crosses represent data from Carlson (Ref. 15), the diamonds data from Samson and Haddad (Ref. 18), and the squares data from Wight and van der Wiel (Ref. 19). The ion yield measurements of Schmidt et al. (Ref. 20) and Holland et al. (Ref. 21) discussed in the text are given by triangles and by stars, respectively. The solid curve shows calculations by Chang and Poe (Ref. 14), whereas the dotted and dash-dotted curves represent dipole lengths and velocity results from Carter and Kelly (Ref. 13). 
values coincide surprisingly well with ion yield measurements performed later using a time-of-flight mass spectrometer, ${ }^{21}$ the reason for this agreement being still unknown. Nevertheless there is clear evidence that the interpretation of double-ionization rates ${ }^{13,14}$ in terms of shake-off processes has to be reconsidered in light of our new results on participator Auger transitions following valence photoionization. In addition to the conceptual importance of such a reconsideration this may also have some impact on the study of threshold laws for double electron ejection, such as Wannier's law, ${ }^{22}$ by multipleionization measurements, as well as on the investigation of the energy and angular distribution of shake-off electrons. Furthermore, it may open new ways for a complete analysis of satellite transitions in a nonrelativistic approach similar to the first studies in this direction on main-line transitions ${ }^{23}$ and could enable scanning Auger spectroscopy to monitor valence-based orbitals or bands at low excitation energies instead of highly excited core levels in a possible future application of the observed effect. We expect to see Auger decay of valence vacancies not only in atoms but also in molecules where we observed the first evidence for such a behavior in $\mathrm{CO}$.

In conclusion, we have observed many previously unexpected low-energy Auger transitions in neon and argon resulting from recombination of valence, particularly inner-valence, vacancies. The mechanism responsible for these transitions is participator Auger decay following photoionization with excitation.

Our results on the shake-off energy distribution confirm theoretical predictions on the symmetric shape of this distribution. The results concerning the Auger decay rates, however, require a reconsideration of valence double-ionization rated being interpreted in terms of and compared to shake-off intensities.

Finally some consequences and possible applications of the observed valence participator Auger transitions were briefly discussed.

We thank Professor Sonntag and the members of his research group for their assistance with the beam line and their interest. We are indebted to Professor Peatman for critical reading of the manuscript. This work was supported by the Bundesminister für Forschung und Technologie under Contract No. 05314 EX B2.
${ }^{1}$ P. Auger, J. Phys. Radium 6, 205 (1925).

${ }^{2}$ W. Mehlhorn, in Atomic Inner-Shell Physics, edited by B. Crasemann, Physics of Atoms and Molecules (Plenum, New York, 1985), p. 119.

${ }^{3}$ T. A. Carlson and M. O. Krause, Phys. Rev. 140, A1057 (1965).

${ }^{4}$ T. Åberg, Phys. Rev. 156, 35 (1967).

${ }^{5}$ U. Gelius, J. Electron Spectrosc. Relat. Phenom. 5, 985 (1974).

${ }^{6}$ M. O. Krause, T. A. Carlson, and W. E. Moddemann, J. Phys. (Paris) Colloq. 32, C4-139 (1971).

${ }^{7}$ H. Kossmann, B. Krässig, V. Schmidt, and J. E. Hansen, Phys. Rev. Lett. 58, 1620 (1987).

${ }^{8}$ S. Svensson, B. Eriksson, N. Märtensson, G. Wendin, and U. Gelius, J. Electron Spectrosc. Relat. Phenom. 47, 327 (1988).

${ }^{9}$ M. G. White, R. A. Rosenberg, G. Gabor, E. D. Poliakoff, G. Thornton, S. H. Southworth, and D. A. Shirley, Rev. Sci. Instrum. 50, 1268 (1979).

${ }^{10}$ U. Becker, R. Hölzel, H. G. Kerkhoff, B. Langer, D. Szostak, and R. Wehiltz, Phys. Rev. Lett. 56, 1120 (1986).

${ }^{11}$ U. Becker, D. Szostak, H. G. Kerkhoff, M. Kupsch, B. Langer, R. Wehlitz, A. Yagishita, and T. Hayaishi, Phys. Rev. A 39, 3902 (1989).

${ }^{12}$ F. Wuilleumier and M. O. Krause, Phys. Rev. A 10, 242 (1974).

${ }^{13}$ S. L. Carter and H. P. Kelly, Phys. Rev. A 16, 1525 (1977).

${ }^{14}$ T. N. Chang and R. T. Poe, Phys. Rev. A 12, 1432 (1975).

${ }^{15}$ T. A. Carlson, Phys. Rev. 156, 142 (1967).

${ }^{16}$ T. N. Chang, T. Ishihara, and R. T. Poe, Phys. Rev. Lett. 27, 838 (1971).

${ }^{17}$ P. A. Heimann, U. Becker, H. G. Kerkhoff, B. Langer, D. Szostak, R. Wehlitz, D. W. Lindle, T. A. Ferrett, and D. A. Shirley, Phys. Rev. A 34, 3782 (1986); P. Lablanquie, thèse d'Etat Université de Paris-Sud, Centre d'Orsay, 1989 (unpublished).

${ }^{18}$ J. A. R. Samson and G. N. Haddad, Phys. Rev. Lett. 33, 875 (1974).

${ }^{19}$ G. R. Wight and M. J. van der Wiel, J. Phys. B 9, 1319 (1976).

${ }^{20}$ V. Schmidt, N. Sandner, H. Kuntzemüller, P. Dhez, F. Wuilleumier, and K. Källne, Phys. Rev. A 13, 1748 (1976).

${ }^{21}$ D. M. P. Holland, K. Codling, J. B. West, and G. V. Marr, J. Phys. B 12, 2465 (1979).

${ }^{22}$ G. H. Wannier, Phys. Rev. 90, 817 (1953).

${ }^{23}$ A. Hausmann, B. Kämmerling, H. Kossmann, and V. Schmidt, Phys. Rev. Lett. 61, 2669 (1988). 